\title{
Tenascin is overexpressed in vitiligo lesional skin and inhibits melanocyte adhesion
}

\author{
I.C.LE POOLE, ${ }^{*} \dagger$ R.M.J.G.J.VAN DEN WIJNGAARD, ${ }^{*} \dagger$ W.WESTERHOF $* \dagger$ AND \\ P.K.DAS $* \dagger$ \\ Departments of *Dermatology and $\dagger$ Pathology, AMC/Amsterdam University, Meibergdreef 9, 1106 AZ Amsterdam, the \\ Netherlands \\ $\ddagger$ Dutch Institute for Pigmentary Disorders (SNIP), Meibergdreef 35, Amsterdam, the Netherlands \\ Accepted for publication 24 February 1997
}

\begin{abstract}
Summary The aetiology of vitiligo remains obscure. In this study, the role of integrins in the observed inability of melanocytes to repopulate lesional skin was investigated. Antibodies directed to $\alpha_{2}, \alpha_{3}, \alpha_{5}, \alpha_{\mathrm{v}} \alpha_{6}, \beta_{1}$ and $\beta_{3}$ integrin subunits were used. Immunohistology revealed no marked differences in the overall levels of expression of integrins between control, non-lesional, perilesional or lesional skin. Moreover, no differences were noted in the level of expression of integrins or the adhesive capacity between cultured control cells derived from three separate donors and vitiligo-derived melanocytes from two donors. Rather, it was clearly observed that towards the lesion, vitiligo skin contains increasing amounts of tenascin in the basal membrane and papillary dermis in five patients employing T2H5 antihuman tenascin antibody. The anti-adhesive effect observed in vitro for this extracellular matrix molecule using normal melanocytes may contribute to loss of pigment cells in vitiligo or to ineffective repopulation of the lesions.
\end{abstract}

In vitiligo, melanocytes are gradually lost in depigmenting lesions of the skin. ${ }^{1}$ It has been suggested that defective adhesion is involved in loss of melanocytes in this pigmentary disorder. ${ }^{2}$ Moreover, the persistence of depigmented lesions is suggestive of defective melanocyte migration which may be reversed by photochemotherapy, ${ }^{2}$ and therefore baseline expression of adhesion molecules may differ in vitiligo as compared with control skin. Melanocyte adhesion, spreading and migration is mediated by integrins. ${ }^{3}$ Employing antibodies to integrin subunits, the specific integrins involved in each process have been identified in vitro. ${ }^{3,4}$

Importantly, all integrins thus far identified on melanocytes can also be found on neighbouring keratinocytes. Although immunohistology has been employed to identify integrins expressed on melanocytes in situ per se, ${ }^{3}$ this may not be the method of choice to determine differences in the level of expression by melanocytes. Even by double immunostaining procedures, it is difficult to distinguish between integrin expression by melanocytes vs. neighbouring keratinocytes. Therefore, in vitro cultures derived from control as well as from vitiligo non-lesional skin were employed to

Correspondence: I.Caroline Le Poole, Department of Dermatology, University of Cincinnati, PO Box 670592, Cincinnati, OH 452670592, U.S.A. investigate further potential differences in adhesive behaviour in vitiligo and control skin. In addition, the use of cultured cells provides the opportunity to perform functional adhesion assays. In this respect it is of great interest to note that cultured melanocytes from nonlesional vitiligo skin appear to retain disease-related characteristics. It has been demonstrated that vitiligo melanocytes have altered growth characteristics under limiting conditions. ${ }^{5}$ Moreover, upon electron microscopic observation, dilated rough endoplasmic reticulum (RER) is apparent in diseased cells from human and mouse vitiligo subjects. ${ }^{6,7}$ Therefore, the use of cultured cells to investigate differences in adhesive characteristics is justified.

The level of expression of relevant integrin subunits by vitiligo melanocytes was compared with baseline expression by control melanocytes. As integrins require activation prior to mediating their respective adhesive functions, ${ }^{8}$ potential differences in intrinsic adhesive behaviour were also investigated. The ability to adhere to different extracellular matrix (ECM) molecules, namely collagen IV, laminin, fibronectin, hyaluronic acid and tenascin was assessed using ${ }^{51} \mathrm{Cr}$ release as a measure of the fraction of cells that have adhered. Results of these experiments illustrate the importance of the composition of the ECM for the adhesive 
behaviour of cells. In this respect, it has been reported that the concentration of tenascin can increase manyfold in pathological conditions. In skin, elevated tenascin content has been observed during tumorigenesis and wound healing. ${ }^{2,9}$ Moreover, others have reported the abundance of this ECM molecule in vitiligo skin in a preliminary investigation, ${ }^{10}$ whereas this ECM molecule may interfere with melanocyte adhesion. ${ }^{11-13}$ Consequently, the tenascin content of the basal membrane in non-lesional, perilesional and lesional vitiligo skin was compared by immunohistology. The combination of functional assays and immunohistological examination provides insight into the role of adhesive mechanisms in the aetiopathology of vitiligo.

\section{Materials and methods}

\section{Skin sections}

Expression of integrin receptors was evaluated in nonlesional, perilesional and lesional limb skin from three female Caucasian patients with progressive $(n=2)$ or stationary vitiligo prior to medication (mean age 34 years) and compared with expression in control foreskin (16 years). Absence of melanocytes in lesional skin was confirmed as described previously ${ }^{1}$ using antibody NKIbeteb. ${ }^{13}$ Four millimetre biopsies were obtained and snap-frozen in liquid nitrogen. Six micrometer frozen sections were fixed for $10 \mathrm{~min}$ in cold acetone and stored at $-20^{\circ} \mathrm{C}$ until use. Similarly, the presence of tenascin was evaluated in non-lesional, perilesional and lesional skin from five generalized vitiligo patients with progressive depigmentation. Biopsies were taken either from the limbs $(n=4)$ or from the trunk. The patient group of four males and one female had a mean age of 45 years and an average duration of 13 years of vitiligo.

\section{Melanocyte culture}

Melanocytes were isolated from blister roof tops ${ }^{14}$ or from surgical specimens ${ }^{15}$ essentially as previously described. The culture medium consisted of Ham's F10 (Gibco Life Technologies, Breda, the Netherlands) with $1 \% \mathrm{v} / \mathrm{v}$ Ultroser $\mathrm{G}$ (Gibco), $10 \mathrm{ng} / \mathrm{mL}$ tetradecanoyl phorbol 13-acetate (Sigma-Aldrich, Zwijndrecht, the Netherlands), $0 \cdot 1 \mathrm{mmol} / \mathrm{L} \quad 3$-isobutyl-methylxanthine (Sigma), $100 \mathrm{IU} / \mathrm{mL}$ penicillin $/ 100 \mathrm{mg} / \mathrm{mL}$ streptomycin (Gibco Life Technologies) and $2 \mathrm{mmol} / \mathrm{L}$ glutamine (Gibco Life Technologies). Contaminating cell types were selectively eliminated by addition of $100 \mu \mathrm{g} / \mathrm{mL}$ geneticin (Gibco Life Technologies) to the culture medium for 3 consecutive days. ${ }^{16}$ Elimination of contaminating cell types was confirmed by phase contrast light microscopy as well as by fluorescence-activated cell scanning (FACS) analysis (see below) using antibody NKI-beteb ${ }^{13,16}$ as the primary antibody. Control melanocyte cultures used in this study were derived from foreskin $(n=2)$ or abdominal skin $(n=1)$ from donors of varying pigmentation with a mean age of 16 years. Vitiligo melanocytes were derived from Caucasian non-lesional limb skin. Patients had a mean age of 38 years at the time of cell isolation.

\section{Immunohistology}

To detect expression of integrin subunits, frozen skin sections were incubated with a primary antibody in predetermined optimal dilution for an hour. Subsequently, peroxidase-labelled rabbit antimouse antiserum (Dakopatts, Glostrup, Denmark) was applied 1:100 for $30 \mathrm{~min}$. To enhance the signal, peroxidase-labelled swine antirabbit antiserum (Dakopatts) was applied 1:50 for $30 \mathrm{~min}$. Finally, aminoethylcarbazole (AEC) (Sigma) dissolved in acetate buffer $\mathrm{pH} 4.9$ was used to reveal the presence of the antigen under study. Nonspecific staining was prevented both by pre-incubation with $10 \%$ normal swine serum and by washing with Tris(hydroxymethyl)aminomethane-buffered saline (TBS) throughout the staining procedure. For the $\alpha_{2}$ and $\alpha_{3}$ integrin subunits, double stainings with antibody NKIbeteb specific for the gp100/Pmel17 protein of melanocytes were performed to demonstrate loss of melanocytes in conjunction with levels of integrin expression.

In the skin, the double-staining procedure was performed as follows. A mixture of primary antibodies in optimal dilutions for single stainings was applied to skin sections for $1 \mathrm{~h}$ at room temperature. After thorough washing, a mixture of secondary antibodies was applied for $1 \mathrm{~h}$ at room temperature. The mixture consisted of peroxidase-labelled goat antimouse IgG2b and alkaline phosphatase-labelled goat antimouse IgG1 ( $1: 20$ each, Southern Biotechnology, Birmingham, AL, U.S.A.). Alkaline phosphatase was localized with Fast Blue BB (Sigma), followed by revealing peroxidase activity using AEC as mentioned under single stainings.

Alternatively, for tenascin single immunostainings skin sections were pretreated with 10\% normal goat serum (Dakopatts) for $15 \mathrm{~min}$ to block aspecific binding of primary antibodies. Primary antibody $\mathrm{T} 2 \mathrm{H} 5^{17}$ was optimally diluted in 10\% normal human serum in TBS. Skin sections were incubated for $1 \mathrm{~h}$ at room temperature with the primary antibody at relevant dilutions 
in TBS. In the second step, mouse monoclonal antibodies were recognized by biotin-labelled $\mathrm{F}(\mathrm{ab}) 2$ rabbitantimouse antiserum (Dakopatts) used 1:200. To improve antigen detection, peroxidase labelled biotin and streptavidin were mixed according to the manufacturer's protocol of a Dakopatts streptABC detection kit. Peroxidase activity was detected as above. Antibodies used for in situ detection of integrin subunits $\alpha_{2},{ }^{18} \alpha_{3},{ }^{19}$ $\alpha_{5}{ }^{20} \alpha_{6}{ }^{21}$ and $\beta_{1}{ }^{22}$ are further described in Table 1. Pre-evaluated optimal antibody concentrations used for immunohistology were on average 2.5 times those used for FACS analysis. Abundance and intensity of staining was evaluated by two investigators independently. The abundance of subepithelial tenascin was determined as the average thickness of the subepithelial layer immunostained by T2H5. Quantification was performed by image analysis using a Zeiss Interactive Digital Analysis System (ZIDAS) (Zeiss, Thornwood, NY, U.S.A.). Abundance was measured as the subepithelial immunostained surface area $\left(\mathrm{mm}^{2}\right)$ divided by length of the dermoepidermal junction $\left(\mathrm{mm}^{2}\right)$.

\section{FACS analysis}

Cultured adherent cells were scraped for three-step indirect immunofluorescent stainings. Cells were incubated with optimally diluted primary antibody in phosphate-buffered saline (PBS) containing 10\% normal human serum and $0 \cdot 01 \%$ azide for $1 \mathrm{~h}$ at $4{ }^{\circ} \mathrm{C}$. Cells were washed in $2 \%$ fetal calf serum (FCS) containing PBS with $0 \cdot 01 \%$ azide. In the second step biotin labelled rabbit antimouse antiserum (Dakopatts) 1:50 was added for $30 \mathrm{~min}$, followed by phycoerythrin labelled streptavidin (Dakopatts) 1:25 for $30 \mathrm{~min}$. Fluorescence was measured in 5000 cells per sample by FACS analysis employing a Becton Dickenson FACScan (Lincoln Park, NJ, U.S.A.) and evaluated by the Becton Dickenson LYSIS II computer program. Anti- bodies directed to integrin subunits $\alpha_{2},{ }^{18} \alpha_{3},{ }^{19} \alpha_{5},{ }^{20}$ $\alpha_{\mathrm{v}},{ }^{23} \alpha_{6},{ }^{21} \beta_{1},{ }^{22} \beta_{3}{ }^{24}$ and $\beta_{4}{ }^{25}$ are described further in Table 1.

\section{Adhesion assays}

Cultured melanocytes from control and vitiligo donors as described under 'melanocyte culture' were used to perform adhesion assays. Adhesion assays were performed as described previously by Danen et al. ${ }^{26}$ and briefly recaptulated below. Ninety-six well flat-bottom microtitre plates were coated with $20 \mu \mathrm{g} / \mathrm{mL}$ of one of the following: human plasma-derived laminin (Sigma), human foreskin fibroblast-derived fibronectin (Sigma), human placental collagen IV (Sigma), rooster combderived hyaluronic acid (Sigma) or human glioblastoma cell line-derived tenascin (Sigma). Wells coated at $4{ }^{\circ} \mathrm{C}$ overnight were washed in PBS containing $0 \cdot 25 \%$ bovine serum albumin (BSA). For assays where the inhibitory effect of tenascin for melanocyte adhesion was to be determined, some wells precoated with fibronectin were subsequently coated with tenascin as mentioned above.

Fibronectin was chosen as a model ECM component to investigate potential inhibition of adhesion by tenascin because melanocytes readily adhered to fibronectin. Therefore, a potential inhibitory effect is clearly detected. Cultured cells were detached using $0.02 \%$ ethylene diamine tetraacetic acid (EDTA) in PBS and labelled with $500 \mu \mathrm{Ci} \mathrm{Na} \mathrm{CrO}_{4}$ in a volume of $100 \mu \mathrm{L}$ Dulbecco's modified Eagle's medium (DMEM) containing $0 \cdot 25 \% \mathrm{BSA}$ for $90 \mathrm{~min}$ at room temperature. Washed cells were allowed to adhere for $75 \mathrm{~min}$ at $2 \times 10^{4}$ cells per well at $37^{\circ} \mathrm{C}$ and $5 \% \mathrm{CO}_{2}$. Non-adherent cells were removed by washing with PBS containing $0 \cdot 25 \%$ BSA. Attached cells were lysed by addition of $1 \%$ Triton-X-100 in PBS. Radioactivity of the supernatant was measured in a gamma counter. Percentage

Table 1. Antibodies to integrins used in this investigation

\begin{tabular}{llcll}
\hline & & & & Source \\
Integrin subunit & Antibody & Reference & & $\begin{array}{c}\text { Dilution } \\
\text { FACS }\end{array}$ \\
\hline$\alpha_{2}$ & $10 \mathrm{G} 11$ & 18 & Dr C.G.Figdor, Nijmegen University, the Netherlands & $1: 25$ \\
$\alpha_{3}$ & J143 & 19 & Dr C.G.Figdor & $1: 50$ \\
$\alpha_{5}$ & SAM1 & 20 & Dr C.G.Figdor & $1: 50$ \\
$\alpha_{\mathrm{V}}$ & NKI-M7 & 23 & Dr C.G.Figdor & $1: 50$ \\
$\alpha_{6}$ & GoH3 & 21 & Dr A.Sonnenberg, NKI, Amsterdam, the Netherlands & $1: 2 \cdot 5$ \\
$\beta_{1}$ & $4 \mathrm{~B} 4$ & 22 & Telios, San Diego, CA, U.S.A. & $1: 100$ \\
$\beta_{3}$ & C17 & 24 & Dr A.Sonnenberg & $1: 25$ \\
$\beta_{4}$ & 3E1 & 25 & Telios & $1: 25$ \\
\hline
\end{tabular}



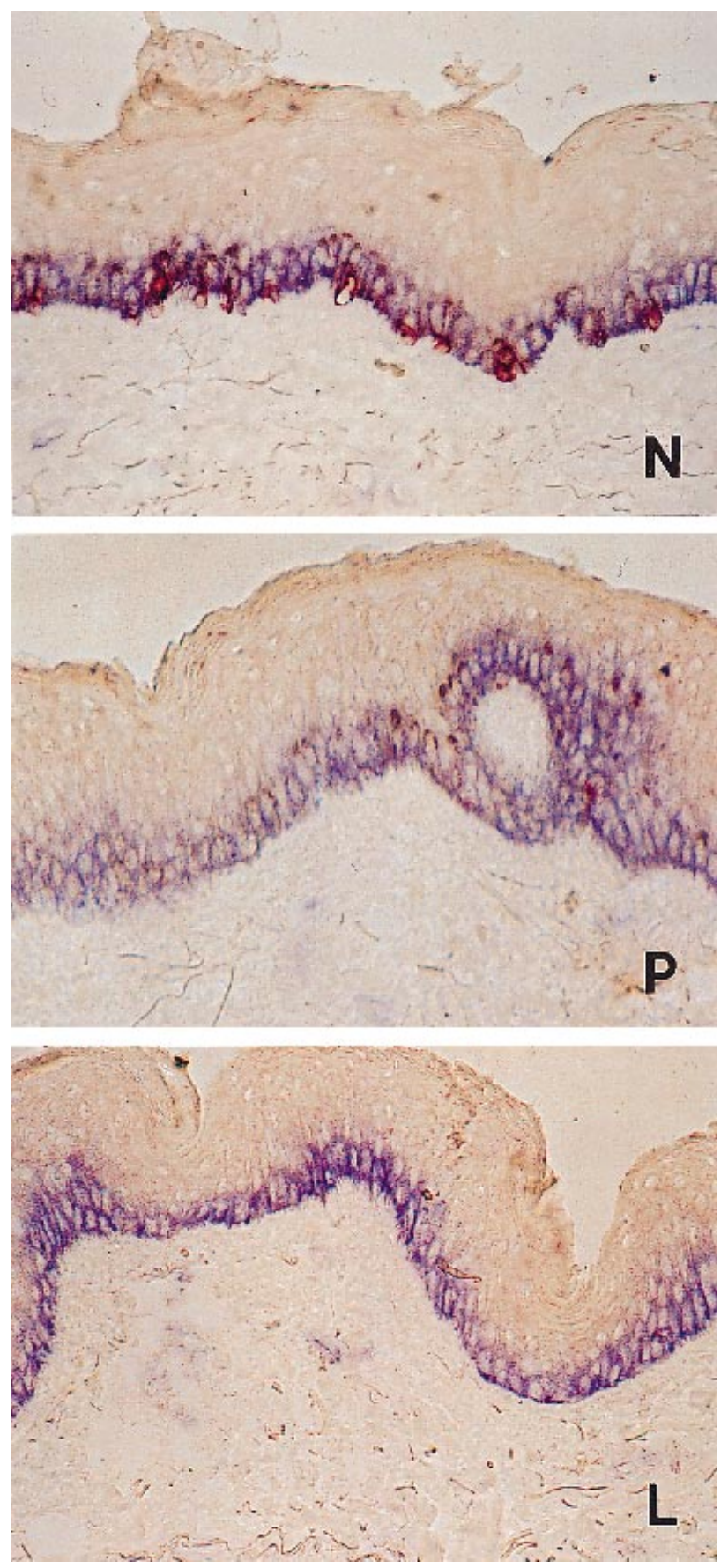

Figure 1. Double immunostaining to detect the expression of the $\alpha_{3}$ integrin subunit by antibody J143 (shown in blue) as well as the presence of melanocytes using the highly specific antibody NKI-beteb (shown in red) in non-lesional $(\mathrm{N})$, perilesional $(\mathrm{P})$ and lesional $(\mathrm{L})$ skin. Loss of melanocytes in lesional skin is apparent. Moreover, the difficulty to differentiate between integrin expression by melanocytes vs. expression by neighbouring keratinocytes is demonstrated. (Original magnification $100 \times$.) adhesion was determined as $100 \times$ the mean value obtained from five wells/total cell count. PBS/BSAcoated wells were used to determine baseline adhesion values.

\section{Results}

\section{Expression of integrin subunits in situ}

Integrin subunits $\alpha_{2}, \alpha_{3}$ and $\alpha_{6}$ were expressed in the basal layer of the epidermis in frozen sections obtained from control skin as well as in sections obtained from non-lesional, perilesional and lesional skin obtained from three vitiligo patients. No in situ expression of $\alpha_{5}$ was observed.

In Fig. 1, a representative $\alpha_{3}$ (blue)/melanocyte (red) double staining is shown. No difference in the level of integrin expression is apparent between non-lesional, perilesional and lesional vitiligo skin. The loss of melanocytes towards lesional skin is represented by loss of NKI-beteb immunostaining in lesional skin. In the perilesional skin from this progressive vitiligo lesion, remnants of melanocytes were observed. Similar results were obtained in $\alpha_{2}$ /melanocyte double stainings. Similarly, with antibodies to $\alpha_{6}$ and $\beta_{1}$ no differences in the levels of integrin expression were observed among non-lesional, perilesional and lesional vitiligo skin.

\section{Integrin expression by cultured melanocytes}

Aberrations in the level of expression of integrins specifically in melanocytes were investigated by FACS analysis. In Fig. 2, baseline expression of integrin subunits is compared between control and vitiligo melanocytes. It must be noted that among antigens, differences in mean

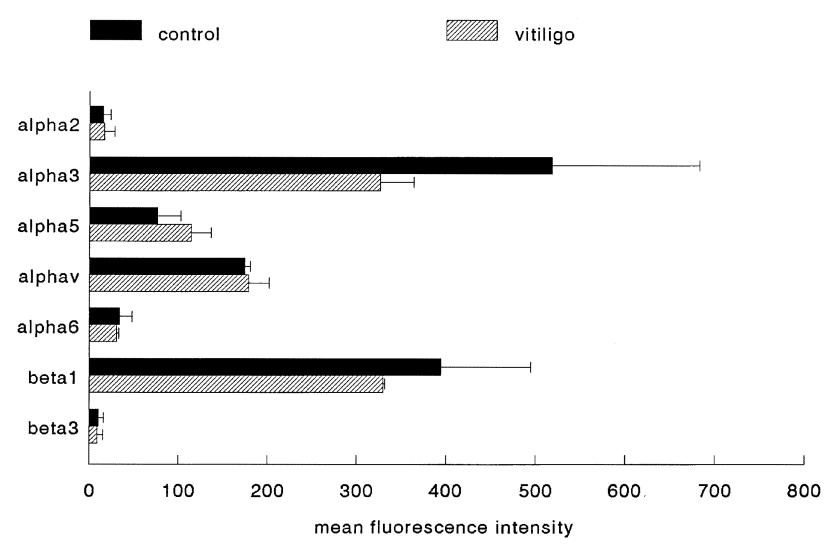

Figure 2. Baseline expression of integrin subunits by control (three cultures) and vitiligo (two cultures) melanocytes, represented as mean fluorescence intensities \pm standard error of the mean (SEM). 


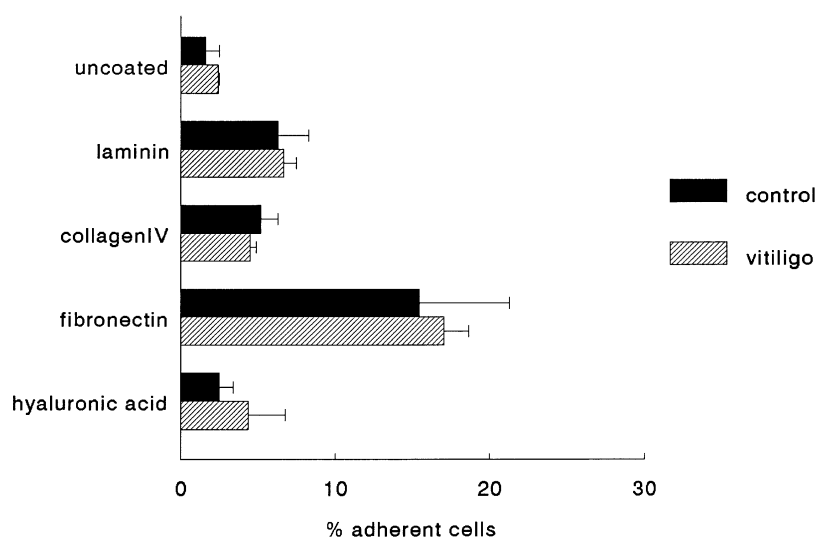

Figure 3. Adhesion to extracellular matrix components by control (three cultures) and vitiligo (two cultures) melanocytes, represented as the percentage of cells that have adhered \pm SEM.

fluorescence intensities may reflect differences in affinity of the antibody for the corresponding antigen. No significant difference in the level of expression of any of the integrin subunits under study was observed upon comparison of control and vitiligo cells.

\section{Adhesion of control and vitiligo melanocytes to extracellular matrix components}

As shown in Fig. 3, no intrinsic difference in adhesive capacity to fibronectin, laminin, collagen IV or hyaluronic acid was observed between vitiligo and control melanocyte cultures. In culture, melanocytes adhere

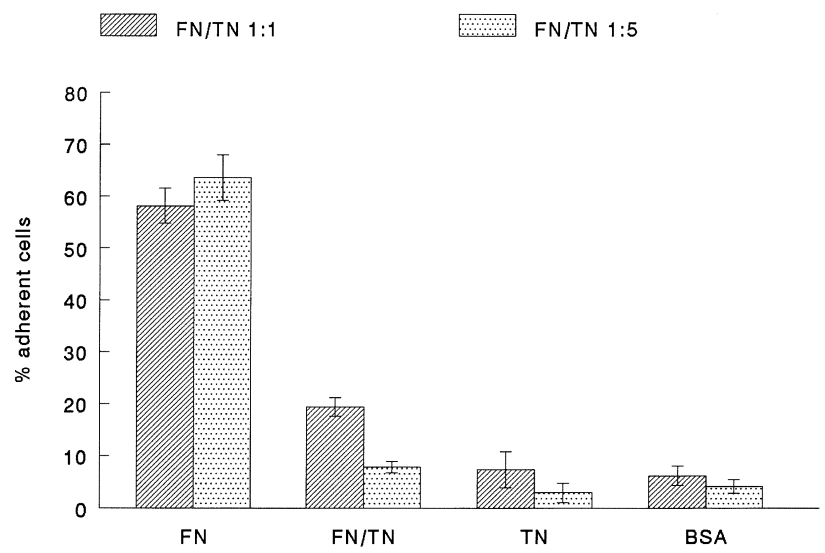

Figure 4. The effect of tenascin on neonatal melanocyte adhesion to fibronectin. Results from two representative experiments, represented as the average percentage of adherent cells in five wells \pm standard deviation (SD). FN: fibronectin-coated wells. FN/TN: wells coated with fibronectin and tenascin. TN: tenascin-coated wells. BSA: mock-coated wells, pretreated with bovine serum albumin. In one experiment, a fibronectin to tenascin ratio of $1: 1 \mathrm{w} / \mathrm{w}$ was used, whereas in the other experiment shown the tenascin concentration used for coating was increased fivefold. most readily to ECM component fibronectin. However, laminin and collagen IV also stimulate adhesion as compared with uncoated plastic. Adhesion to hyaluronic acid-coated plastic was comparable with that observed to uncoated plastic. In Fig. 4, the adhesive capacity of melanocytes to fibronectin in the presence and absence of tenascin is compared at two different tenascin concentrations. Evidently, tenascin inhibits adhesion of melanocytes to fibronectin to an extent increasing with rising tenascin concentration. Importantly, percentages of adherent cells can vary per experiment as a function of several experimental variables beyond the scope of this investigation. Thus, results are best compared within experiments.

\section{Tenascin in vitiligo skin}

In Fig. 5, the average thickness of the tenascin-containing layer beneath the epidermis is representative of the abundance of tenascin in control vs. vitiligo skin. Interestingly, in all progressive vitiligo patients, the abundance of tenascin increases towards the lesion. Moreover, the increase in tenascin abundancy tends to be inversely correlated to the duration of the disease. Figure 6 shows a representative example of $\mathrm{T} 2 \mathrm{H} 5$ immunostaining in nonlesional, perilesional and lesional skin from a vitiligo patient, demonstrating an increase in tenascin content of the papillary dermis towards the lesion.

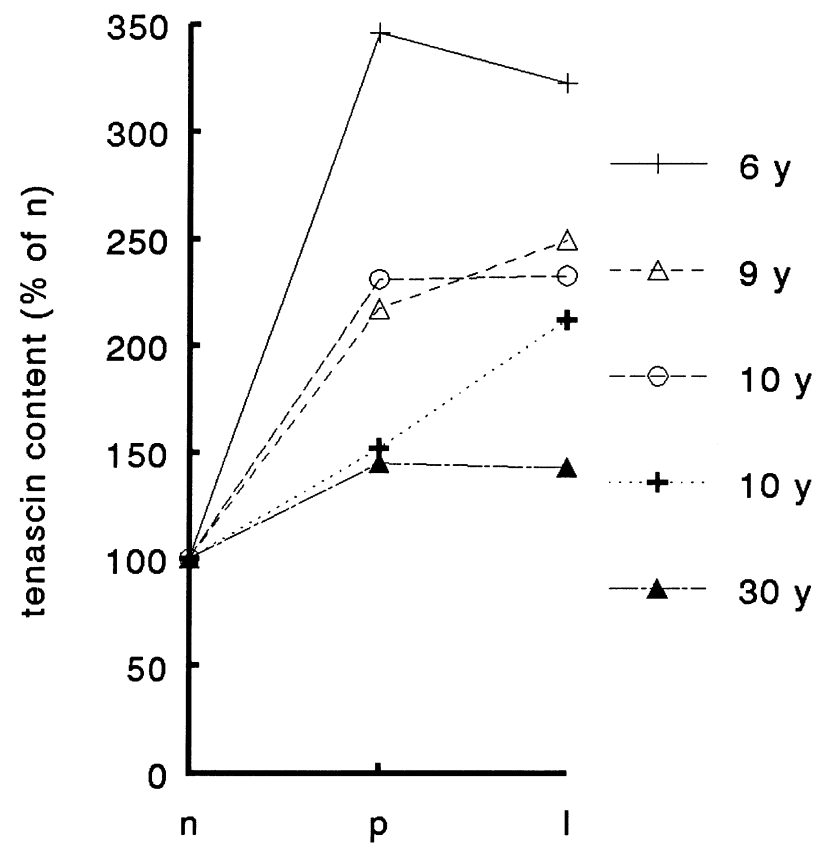

Figure 5. Quantification of tenascin in non-lesional (n), perilesional (p) and lesional (l) vitiligo skin relative to expression in non-lesional skin. The duration of vitiligo is noted for the patients under study. 

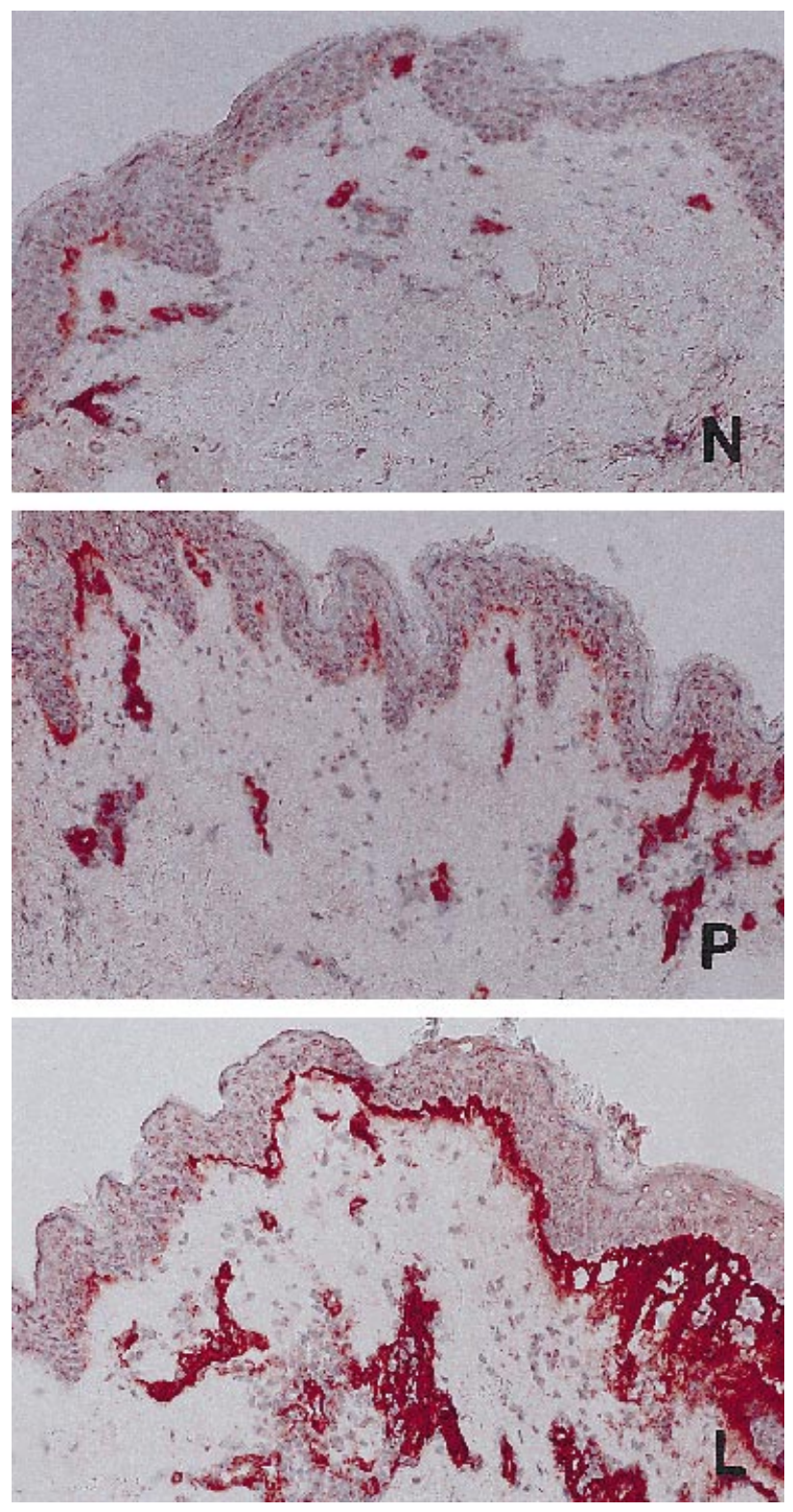

Figure 6. Tenascin expression in non-lesional $(\mathrm{N})$, perilesional $(\mathrm{P})$ and lesional (L) vitiligo skin, revealed by $\mathrm{T} 2 \mathrm{H} 5$ immunostaining (original magnification $50 \times$ ).

\section{Discussion}

From this investigation it can be concluded that there is no overall difference in the level of expression of integrins at the cell surface of non-lesional vitiligo and control melanocytes. In addition, functional studies did not reveal any significant difference in adhesive capacity between non-lesional vitiligo melanocytes vs. melanocytes derived from healthy control skin. These findings are in accordance with the fact that no difference was observed in migratory capacity of melanocytes in the epibolic outgrowth of explants cultured from non-lesional vs. control human skin. ${ }^{27}$ As no intrinsic aberrations were observed in the function of integrins on the vitiligo melanocyte cell surface, it follows that conclusions drawn from adhesion and migration studies performed employing control melanocytes will also apply to melanocytes derived from vitiligo patients.

This is of major importance as for repigmentation, melanocytes surrounding vitiligo lesions and melanocytes from hair shafts ${ }^{28}$ need be stimulated to re-enter vitiligo lesional skin. Observations that LTC-4 and nerve growth factor (NGF) stimulate melanocyte migration ${ }^{29}$ are, thus, of great interest for the development of new therapeutic modalities. However, results presented here concerning the abundance of tenascin in vitiligo skin also indicate that vitiligo lesions present a hostile environment for melanocytes. Tenascin has been described as an anti-adhesive molecule which is explained at least in part by its ability to bind and/or shield molecules otherwise mediating adhesion of several cell types. ${ }^{30-32}$ The presence of tenascin has been suggested to augment dispersion of the malignant derivative of melanocytes, thereby contributing to metastasis of malignant melanomas. ${ }^{9}$ In this study it was observed that vitiligo patients exhibited an increase in tenascin content of the ECM in contact with epidermal melanocytes. In addition, it was shown that tenascin markedly inhibits adhesion of melanocytes to fibronectin. Interestingly, as it has been reported that the composition of the ECN can influence melanogenesis, ${ }^{33}$ it appears that other aspects of melanocytes in addition to adhesion may be affected by the observed increase in tenascin content.

It is yet to be determined whether the observed tenascin increase in vitiliginous skin precedes loss of melanocytes or occurs as a consequence of local immune reactivity. Cytokines produced as a consequence of local immune reactivity may contribute to an accumulation of tenascin. Evidence is accumulating to support the role that cell-mediated immunity is involved in the aetiopathogenesis of vitiligo. ${ }^{35,36}$ This is most overtly observed in inflammatory vitiligo. ${ }^{37} \mathrm{In}$ this respect it is of interest to note that, in inflammatory vitiligo patients, the tenascin increase is reportedly most marked within the inflammatory rim, whereas in generalized vitiligo patients, the increase can be greatest in the lesional area. This suggests that tenascin induction is a relatively late event and may interfere with repigmentation rather than induce depigmentation. The cell type responsible for the observed overproduction remains obscure. In this respect it is of interest that 
elevated concentrations of transforming growth factor- $\beta$ (TGF- $\beta$ ) can induce tenascin synthesis in fibroblasts. ${ }^{37}$ However, tenascin produced by fibroblasts would be likely to result in overproduction in the reticular dermis rather than in the basement membrane. It appears to be highly unlikely that melanocytes are responsible for the observed increase, as the highest tenascin content is observed in lesional skin, where melanocytes are absent. ${ }^{1}$ Moreover, using melanocytes cultured from several donors, no tenascin production was observed by Western blotting, ELISA assays or immunohistology (results not shown). This is in accordance with observations reported by Herlyn et al. concerning a lack of tenascin production by melanocytes. ${ }^{9}$ Considering the location of the increased tenascin content observed in vitiligo, keratinocytes constitute the most likely source of tenascin production in vitiligo perilesional and lesional skin. This is supported by the observation that keratinocytes are capable of tenascin synthesis. ${ }^{38-40}$ It can, therefore, be speculated that the data presented here are in accordance with the hypothesis that keratinocytes are involved in the aetiopathologesis of vitiligo.

\section{Acknowledgments}

Authors wish to acknowledge financial support by Stiefel Laboratories, U.K. and the Vitiligo Foundation, U.S.A. for this investigation. Helpful discussion and donation of materials by Drs A.Sonnenberg, C.G.Figdor, E.R.Boissy, M.Latijnhouwers and J.Schalkwijk are greatly appreciated.

\section{References}

1 Le Poole IC, van den Wijngaard RMJGJ, Westerhof W et al. Presence or absence of melanocytes in vitiligo lesions: an immunohistochemical investigation. J Invest Dermatol 1993; 100: 816-22.

2 Morelli JG, Kincannon J, Yohn JJ et al. Influence of inflammatory mediators and cytokines on human melanocyte function. J Invest Dermatol 1992; 98: 290-5.

3 Hara M, Yaar M, Tang A et al. Role of integrins in melanocyte attachment and dendricity. J Cell Sci 1994; 107: 2739-48.

4 Morelli JG, Yohn JJ, Zekman T, Norris DA. Melanocyte movement in vitro: role of matrix proteins and integrin receptors. J Invest Dermatol 1993; 101: 605-8.

5 Puri N, Phil M, Mojamdar M, Ramaiah A. In vitro growth characteristics of melanocytes obtained from adult normal and vitiligo subjects. J Invest Dermatol 1987; 88: 434-8.

6 Boissy RE, Liu Y, Medrano EE, Nordlund JJ. Structural aberration of the endoplasmic reticulum and melanosome compartmentalization in long-term cultures from vitiligo patients. J Invest Dermatol 1991; 97: 395-404.

7 Boissy RE, Beato KE, Nordlund JJ. Dilated rough endoplasmic reticulum and premature death in melanocytes cultured from the vitiligo mouse. Am J Pathol 1991; 138: 1511-25.

8 Shimizu Y, van Seventer GA, Horgan KJ, Shaw S. Regulated expression and binding of three VLA $\left(\beta_{1}\right)$ integrin receptors on T cells. Nature 1990; 345: 250-3.

9 Herlyn M, Graeven U, Speicher D et al. Characterization of tenascin secreted by human melanoma cells. Cancer Res 1991; 51: $4853-8$.

10 O'Donnel DB, Saddler M, Aronson PJ, Sikha MVKN. Tenascin is abundant in the skin of vitiligo patients. J Invest Dermatol 1992; 98: 620 (Abstr.).

11 Chiquet-Ehrismann R, Kalla P, Pearson CA et al. Tenascin interferes with fibronectin action. Cell 1988; 53: 383-90.

12 Lightner VA, Erickson HP. Binding of hexabrachion (tenascin) to the extracellular matrix and substratum and its effect on cell adhesion. J Cell Sci 1990; 95: 263-77.

13 Vennegoor C, Hageman P, van Nouhuys $\mathrm{H}$ et al. A monoclonal antibody specific for cells of the melanocyte lineage. Am J Pathol 1988; 130: 179-92.

14 Tomita Y, Yamamoto H, Sato C et al. Efficient culturing of human melanocytes from suction blisters. Tokohu J Exp Med 1985; 147: 219-20.

15 Eisinger M, Marko S. Selective proliferation of normal human melanocytes in vitro in the presence of phorbol ester and cholera toxin. Proc Natl Acad Sci USA 1982; 79: 2018-22.

16 Halaban R, Alfano FD. Selective elimination of fibroblasts from cultures of normal human melanocytes. In Vitro 1984; 20: $447-50$

17 Verstraeten AA, Mackie EJ, Hageman PC et al. Tenascin expression in basal cell carcinoma. Br J Dermatol 1992; 127: 571-4.

18 Gittay JC, Brinkman HJM, Modderman PW et al. Human vascular endothelial cells express a membrane protein complex immunochemically indistinguishable from the platelet VLA-2 (glycoprotein Ia-IIa) complex. Blood 1989; 73: 1235-41.

19 Fraded J, Cordon-Cardo C, Thomson T et al. Cell surface antigens of human bladder cancer defined by mouse monoclonal antibodies. Proc Natl Acad Sci USA 1984; 81: 224-8.

20 Keizer GD, te Velde AA, Schwarting R et al. Role of p150,50 in adhesion, chemotaxis and phagocytosis of human monocytes. Eur J Immunol 1987; 17: 1317-22.

21 Sonnenberg A, Janssen H, Hogervorst $\mathrm{F}$ et al. A complex of platelet glycoproteins Ic and IIa defined by a rat monoclonal antibody. J Biol Chem 1987; 264: 13745-50.

22 Morimoto C, Letvin NL, Boyd AW et al. The isolation and characterization of the human helper inducer T cell subset. J Immunol 1985; 134: 3762-9.

23 De Vries JE, Keizer GD, te Velde AA et al. Characterization of melanoma-associated surface antigens involved in the adhesion and motility of human melanoma cells. Int J Cancer 1986; 38: 465-73.

24 Tetteroo PAT, Lansdorp PM, Leeksma OC, von dem Borne AEG. Monoclonal antibodies against human platelet glycoprotein IIIa. Br J Haematol 1983; 55: 509-51.

25 Hessle H, Sakai LY, Hollister DW et al. Basement membrane diversity detected by monoclonal antibodies. Differentiation 1984; 26: $49-54$.

26 Danen EHJ, van Muyen GNP, van de Wiel-van Kemenade E et al. Regulation of integrin-mediated adhesion to laminin and collagen in human melanocytes and in non-metastatic and highly metastatic human melanoma cells. Int J Cancer 1993; 54: 315-21.

27 Le Poole IC, van den Wijngaard RMJGJ, Westerhof $\mathrm{W}$ et al. Organotypic culture of human skin to study melanocyte migration. Pigment Cell Res 1994; 7: 33-43. 
28 Cui J, Shen J, Wang G. Role of hair follicles in the repigmentation of vitiligo. J Invest Dermatol 1991; 97: 410-16.

29 Morelli JG, Kincannon J, Yohn JJ et al. Leukotriene $\mathrm{C}_{4}$ and TGFalpha are stimulators of human melanocyte migration in vitro. J Invest Dermatol 1992; 98: 290-5.

30 Pesheva P, Probstmeier R, Skubitz APN et al. Tenascin-R (J1 160/ 180) inhibits fibronectin-mediated cell adhesion-functional relatedness to tenascin-C. J Cell Sci 1994; 107: 2323-33.

31 Lightner GA, Erickson HP. Binding of hexabrachion (tenascin) to the extracellular matrix and substratum and its effect on cell adhesion. J Cell Sci 1990; 95: 263-77.

32 Chiquet-Ehrismann R, Kalla P, Pearson CA et al. Tenascin interferes with fibronectin action. Cell 1988; 53: 383-90.

33 Buffey JA, Messenger AG, Taylor M et al. Extracellular matrix derived from hair and skin fibroblasts stimulates human skin melanocyte tyrosinase activity. Br J Dermatol 1994; 131: 836-42.

34 Hann SK, Park YK, Chung KY et al. Peripheral blood lymphocyte imbalance in Koreans with active vitiligo. Int J Dermatol 1993; 32: 286-6.
35 Abdel-Nasr MB, Kruger-Krasagakes S, Krasagakis et al. Further evidence for the involvement of both cell mediated and humoral immunity in generalized vitiligo. Pigment Cell Res 1994; 1: 1-8.

36 Le Poole IC, van den Wijngaard RMJGJ, Westerhof W, Das PK. Presence of T cells and macrophages in inflammatory vitiligo skin parallels melanocyte disappearance. Am J Pathol 1996; 4: 121928.

37 Pearson CA, Pearson D, Shibahara S et al. Tenascin: cDNA cloning and induction by TGF- $\beta$. ЕMBO J 1988; 7: 2977-81.

38 Latijnhouwers M, Bergers M, Ponec M et al. Human epidermal keratinocytes are a source of tenascin-C during wound healing. J Invest Dermatol 1997; 108: 776-83.

39 Latijnhouwers M, Bergers M, Ponec M et al. Epidermal keratinocytes are a source of tenascin. In: XVth FECTS Meeting 1996; 112. (Abstr.).

40 Lightner VA. Tenascin: does it play a role in epidermal morphogenesis and homeostasis? J Invest Dermatol 1994; 102: 273-7. 